\title{
Performance of MAC protocols in beaconing Mobile Ad-hoc Multibroadcast Networks
}

\author{
Cristina Rico Garcia, Andreas Lehner, Patrick Robertson, Thomas Strang \\ German Aerospace Center (DLR), Institute for Communications and Navigation
}

\begin{abstract}
The investigation of infrastructureless safety applications in different transportation systems is a hot research topic. The nodes in the network are designed to advertise to the rest of the nodes informationabout the current traffic situation by means of short beacon messages containing speed, direction, positions and other relevant safety information. The scheduling should be organized by the MAC layer so that the transmitted messages arrive successfully as soon as possible at the receiver. The networks that support these kind of applications are Mobile Ad-hoc Multibroadcast Networks (MAMNETs). In this paper we present the challenges the MAC layer for MAMNETs should overcome. We discuss the most important performance metrics of the MAC layers in order to obtain a system independent analysis and show a survey of the factors that may influence the behavior of the MAC layers.
\end{abstract}

\section{Introduction}

A family of new transportation related safety systems is being extensively investigated in the last years. These new safety systems share the same basic principles: The nodes in the network which can be any kind of vehicle, pedestrians or any moving entity, must be constantly aware of the situation in their surroundings in order to avoid dangerous constellations. To do so, they do not rely on previously installed infrastructure. Instead, they periodically broadcast a short beacon containing important safety information like position, speed, direction, etc.

We call the kind of networks that support this type of safety systems beaconing Mobile Ad-hoc Multibroadcast Networks (B-MAMNETs). The term multibroadcast stresses that, in contrast to traditional broadcast networks, where a fixed number of nodes are broadcast transmitters and the rest of the nodes in the network are receivers, in multibroadcast networks all nodes are broadcast transmitters and receivers.

Typical MAMNETs are given in : Car-to-Car Communications (Car2Car) [6] for automotive transportation; Automatic Dependent Surveillance Broadcast systems (ADS-B) [2] for air transportation; Railway Collision Avoidance Systems (RCAS) [13] for railway transportation; Automatic Identification Systems (AIS) [3] for maritime transportation, as well as various military applications. 
For the future, we can foresee many promising applications in the natural disaster management communication systems area when communication cannot rely on infrastructure any more, like monitoring of rescue teams in earthquake or tsunami scenarios.

The performance of these systems and therefore the safety enhancement they can offer, depends directly on the successful reception of the beacons. Here, the Medium Access Control (MAC) layer plays an important role.

Traditionally, the IEEE 802.11 protocol is used for mobile ad-hoc networks (MANETs). However, the fact that the safety systems are working in a multibroadcast beaconing MAMNET network, introduces several challenges in the design of the MAC layer. First of all, the multibroadcast communication mode prevents the usage of any acknowledgment (ACK) packet, as well as the RTS/CTS protocol, both used in the IEEE 802.11 standard. Secondly, the vehicles in the network are moving quickly, so that the topology is changing continuously and the MAC layer should be able to "follow" these changes. And thirdly, since we are dealing with a safety system the delay of the messages must be minimized.

Consequently, the basic assumptions underlying the IEEE 802.11 that were responsible for its success are no longer present for MAMNETs. Therefore, the performance of IEEE 802.11p [12], i.e., the IEEE 802.11 version for MAMNETs, as well as other protocols for MAMNETs must be investigated in detail from an application independent point of view. In this way, the performance of the protocols can be easily mapped to any present or future system.

The first contribution of this paper is the identification of the factors that influence the behavior of the MAC layers in MAMNETs. The second contribution is the establishment of the performance criteria that should be followed in MAMNETs. These criteria must be application independent and useable for any future MAMNET system. We will introduce the Update Delay as the most relevant performance criterion in MAMNETs, since it indicates the capability of the nodes to react to dangerous situations.

The remainder of this paper is organized as follows: Section 2 shows the evolution of wireless networks and the new challenges introduced. In section 3 we discuss influence factors of the MAC layers in MAMNETs. In section 4 the performance criteria of MAMNETs will be analyzed. Finally, section 5 provides the summary.

\section{General network classification}

The design of a MAC layer should be adapted for the kind of network where it will be used. In the literature there are different surveys of MAC layers that can be used for wireless networks [8], for ad-hoc networks [10] and for mobile ad-hoc networks [16].

This section gives an overview about different types of wireless networks and introduces the mobile ad-hoc multibroadcast networks. Figure 1 depicts our classification of the different wireless networks. 


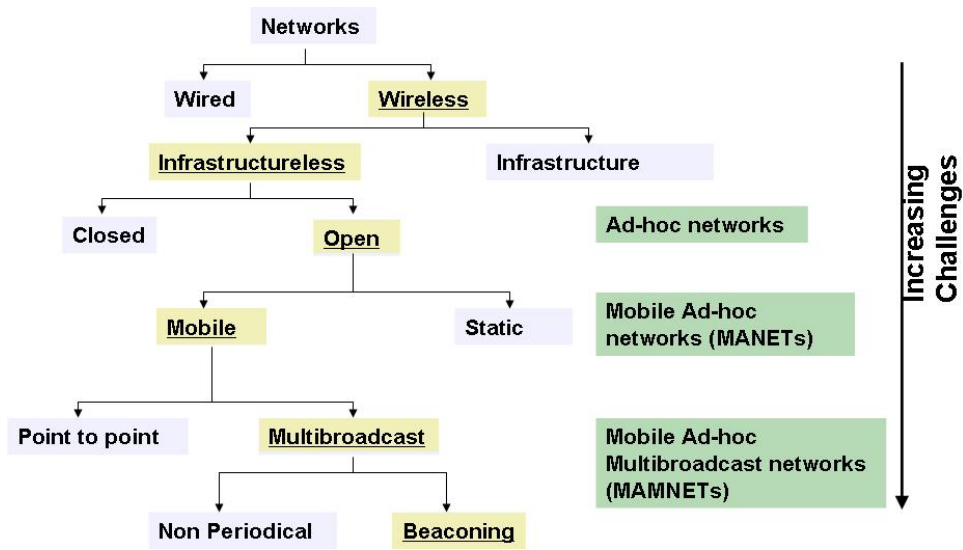

Fig. 1. Network classification

Closed wireless network vs. open wireless network : A closed wireless network is defined as being a wireless network where all the nodes are in range of each other. This means, the nodes are able to receive a message from any another node in the network, no matter how far away it is. On the other hand, in an open wireless network, the network area extends further than the range of the nodes and therefore nodes which are far away are not able to receive each other. In an open network, the hidden terminal problem arises, which is a major design issue for MAC layers. Note a MAC layer always defines no more than a singlehop network since multi-hop is a task of upper layers. Therefore, a single-hoe network might be closed or open.

Ad-hoc network: An Ad-hoc network is a wireless network working in a decentralized mode. This means, there is no infrastructure in charge of coordinating the traffic of the network. The nodes may enter or leave the network suddenly and they must organize themselves in a distributed way so that collisions among messages are avoided.

Mobile Ad-hoc network: A MANET is an ad-hoc network where the nodes can move. The main challenge of this kind of network is that there is no knowledge of how many nodes are in the network and where they are. Therefore, the MAC layer should be able to "follow" the changes experienced in the network.

Multibroadcast Mobile Ad-hoc network: A MAMNET is a MANET where each node is a broadcast station. The multibroadcast communication mode prevents the usage of any acknowledgment (ACK) packet, as well as the RTS/CTS protocol. First, because the broadcasting node does not know from how many nodes ACKs should be received as there is no knowledge about the state of the network. Second, because, the ACKs sent by all receivers would produce collisions among them. Beaconing MAMNETs are a special case of MAMNETs where the messages are short beacons broadcasted with a fixed periodicity. The length of an ACK and a beacon does not differ substantially, therefore, the relative MAC overhead is too costly. 
Obviously, MAMNETs introduce new challenges when designing a suitable MAC protocol. Various MAC layer protocols have been proposed for MAMNETs $[9],[15],[5],[4]$. However, since there is neither a consensus about the necessary metrics in these kinds of networks nor about the MAC performance measurement criteria, there is no way of comparing the performance of the different MAC layers. In the next section, the performance criteria for the analysis of MAC layers in MAMNETs will be discussed.

\section{Factors influencing MAC performance in MAMNETs}

In this section the influencing factors that determine the performance of the layers in MAMNETs are analyzed. In order to study the performance of a MAC layer for a specific system, the influencing factor of the system should be extracted and mapped to the performance criteria curves of a MAC layer, e.g speed and range to node dynamics.

Protocol parameters: The parameters of MAC layer protocols clearly have an influence on the behavior of a MAC layer protocol. Examples of these parameters are the backoff windows in the IEEE 802.11p or the SI parameter in the SOTDMA protocol [9].

Frame duration: An important parameter in MAMNETs is the frame length. A frame duration is defined as the average time between two consecutive transmissions sent by a node. It defines the minimum time the system needs to update the information coming from all the nodes in the network. Typical frame duration values range from $0.5 \mathrm{~s}$ to $2 \mathrm{~min}[6],[3]$.

Closed/open network: A closed or open network has an influence on the MAC layer performance for all those protocols which have to listen to the medium since in open networks the hidden terminal problem arises. The hidden terminal problem is responsible for strong degradation of the performance of MAC layers.

\subsection{Net channel load}

It is widely believed that the net channel load is the most important influencing factor in the performance of a MAC layer [1]. Its relevance has been recognized by the $\mathrm{C} 2 \mathrm{C}-\mathrm{CC}$ community as well [5]. The net channel load or offered traffic is defined as the fraction of a frame occupied by data, i.e. payload. A net channel load of $100 \%$ would be given when there are so many nodes in the network that if they were perfectly synchronized so that they would transmit the data packets one after each other, they would produce neither collisions nor free spaces between data packets. Figure 2 shows a configuration where the number of data packets gives a $100 \%$ net channel load. The net channel load is related to the amount of data transmitted within a frame and not the order in which they are transmitted, so that the same total number of packets transmitted as in Figure 2 , but all at the same time will produce $100 \%$ channel load, too. Please, note that the offered traffic can be larger than $100 \%$ as shown in Figure 8. 


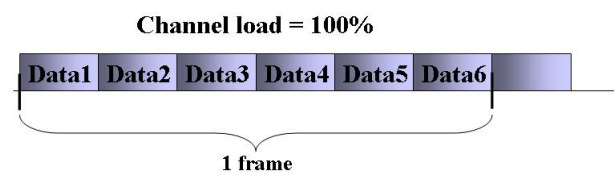

Fig. 2. An example of a frame with $100 \%$ Channel Load

It must be noted that in the calculation of the channel load, only the data bits in the packet should be counted. I.e. neither MAC layer header, nor the backoff time or other waiting times introduced by the MAC layer should be taken into account as part of the offered traffic. This ensures fairness when comparing the performance of different MAC layers.

A MAC layer that requires a larger MAC header could probably deliver a better performance than a MAC layer that does not introduce any header to the data, since it has more valuable information for the MAC layer. However, in order to support the same number of nodes in the network, in the first case the system would need a higher bandwidth than in the second case. The MAC layers must always be compared assuming the same system where only the MAC layer is changed.

The C2C-CC White Paper on Network Design Limits [5] recognizes the channel load as the most relevant network design limit and gives the following definition: The fraction of time that the channel is sensed busy. The problem with this definition is that when the header bits are being transmitted, the channel will be sensed as busy, and as stated above, the header should not be computed in the channel load.

Several publications use the number of nodes instead of the channel load in the network as an influencing factor in the analysis of MAC layer performance [7]. However, this is a complicated parameter to manage as influencing factor. Since different system parameter constellations with the same number of nodes deliver different performances and on the other side, different system parameter constellations with different nodes can deliver the same performance. To overcome this problem, the system parameters can be mapped to the a net channel load in the following way:

$$
\operatorname{Load}(\%)=\frac{\text { Datapackets } / \text { frame }}{\text { Load }_{\max }},
$$

where Load $_{\text {max }}$ tells us how many packets are in the system under $100 \%$ net channel load. The calculation of this factor is performed in a different way depending on whether the network is closed or open.

Net channel load in a closed network : The $100 \%$ net channel load in a closed network is given by

$$
\text { Load }_{\text {max closed }}=\frac{\text { frame }}{\text { Data }_{\text {time }}},
$$

where the frame is in seconds and the Datatime is the length in seconds of a packet calculated as Datatime $=$ Data $_{\text {bits }} \cdot t x_{\text {rate }}$. The transmission rate $t x_{\text {rate }}$ must be calculated computing the whole available bandwidth for the MAC layer. 
A MAC layer that separates its bandwidth in two channels, for example one for MAC control data and another one for data will normally have a better performance in the data channel than a protocol that only uses the data channel without any control channel. Note that in both cases the data transmission rate is the same although the total available bandwidth is larger in the first case. Therefore, again for the sake of fairness in the comparison of different MAC layers the transmission rate should be derived from the available bandwidth for the complete MAC layer and not only from the data bandwidth.

Net channel load in a open network : The $100 \%$ net channel load in a open network is given by

$$
\operatorname{Load}_{\text {max }}=\text { Area }_{\text {network }} \cdot \text { density }_{\text {max }}
$$

where the density max $_{\text {max }}=\frac{\text { Load }_{\text {max }} \text { closed }}{\text { Area }}$. The Areange Arange $_{\text {ran }}$ is the area around a node where its transmissions are received, i.e the communication range area.

Payload/header length: The MAC layer header usually has a fixed length independent of the payload length. A system with a high payload/header rate will be able to attain a bigger channel load than a system with a low payload/header rate. Although MAMNETs systems transmit in general very short messages of around 200 bits, other payload/header rates should be taken into account as influencing factor.

\section{$3.2 \quad$ Topological network dynamic}

We define the topological network dynamic $\delta$ as the quotient of maximum node velocity $v_{\max }$ and minimal communication range Range $\min _{\min }$. I.e. $\delta=\frac{v_{\max }}{\text { Range } \min }$. The influence factor $\delta$ indicates how instable the network can be.

\begin{tabular}{|l||c|c|c|}
\hline Transport system & Min. comm. range & Maximum velocity & $\begin{array}{c}\text { Topological } \\
\text { network dynamic }\end{array}$ \\
\hline \hline Ships - AIS & $40 \mathrm{~km}$ & $60 \mathrm{~km} / \mathrm{h}$ & $1,5 \mathrm{~h}^{-1}$ \\
\hline Airplanes - ADSB & $56 \mathrm{~km}$ & $1000 \mathrm{~km} / \mathrm{h}$ & $16 \mathrm{~h}^{-1}$ \\
\hline Trains - RCAS & $5 \mathrm{~km}$ & $200 \mathrm{~km} / \mathrm{h}$ & $40 \mathrm{~h}^{-1}$ \\
\hline Cars - C2C & $1 \mathrm{~km}$ & $140 \mathrm{~km} / \mathrm{h}$ & $140 \mathrm{~h}^{-1}$ \\
\hline
\end{tabular}

Table 1. Topological network dynamic factor for different systems

traffic model: The traffic model plays an important role. It indicates how the nodes are moving altogether. A network may have a high topological network dynamic factor but if the traffic model is a uniform and parallel movement of the nodes, then the influence of the factor will be lower than if the traffic model is a random and independent movement of the nodes. 


\subsection{Radio channel}

The last influencing factor listed in this paper is the radio channel. The MAC layers should be tested under the conditions of different path loss radio channels, since the influence of the radio channel on the performance of the MAC layer is remarkable. Important radio channels are for example the ideal channel, i.e, the signal is not attenuated in the communication range and afterwards it is

zero and the logarithmic path loss channels. In order to study the influence of the interference of nodes beyond the communication range, a logarithmic path loss channel can be compared to a logarithmic-ideal channel, i.e., a channel with a logarithmic profile in the communication range but with infinite attenuation afterwards.

The Bit Error Rate (BER) might as well have an influence in the performance of the MAC layer for MAC protocols if these need to decode the receiver messages in order to take decisions.

\section{Performance criteria and metrics for the analysis of MAC layers in MAMNETs}

In this section the performance criteria to measure the behavior of the MAC protocols will be discussed. The analysis of the MAC layers should be accomplished in such a way that performance criteria are studied for the different influencing factors listed in the previous section.

\subsection{Update Delay}

In classical ad-hoc networks, the transmission delay is an important performance criterion, specially for real time applications. However it has low practical relevance in MAMNETs.

Transmission Process in ad-hoc Networks: The transmitter wants to send a packet. This packet can be short or long, data, video, sound, etc. The packet is delivered in the buffer queue, and waits there until the MAC layer allows its transmission. Additionally to the delay in the buffer, the delay due to the distance between sender and receiver should be added. Then, the transmitter waits for an acknowledgement from the receiver. In case no acknowledgement is received, the transmitter resends the packet again until it receives a valid acknowledgement. The total transmission delay $T D$ is:

$$
T D=R \cdot\left(Q+t_{c}+t_{a c k}\right)
$$

where $R$ is the number of transmission per single packet necessary for successful reception, $Q$ is the time spent in the buffer queue and other MAC layer waiting time, $t_{c}$ is the transmission time due to the distance between transmitter and receiver and $t_{a c k}$ is the time the transmitter should wait for an acknowledgement before trying a retransmission. 
Transmission Process in MAMNETs: The transmitter is continuously getting information from its onboard localization unit and wants to broadcast a packet every period of time $T$. This packet is a short beacon, which contains updated safety information like the node position, speed, etc. The packet is delivered in the buffer queue. If time $T$ goes by before the MAC layer has ordered transmission of this packet, a new beacon arrives in the queue and replaces the old undelivered beacon since the status information it contains is outdated. The transmitter does not wait for an acknowledgement. On the one hand, in a multibroadcast network acknowledgements are not feasible and on the other hand, there is no sense in retransmitting outdated information, in case no acknowledgement would be received - in particular when the transmitter has more recent status information to send. The total transmission delay $T D$ is:

$$
T D=\left(Q+t_{c}\right)
$$

Obviously the transmission delay in a MAMNET is negligible for the MAC functionality compared to the transmission delay in a standard ad-hoc network and has no practical relevance in the MAC design.

The moving nodes in a MAMNET take decisions and react according to the received update status beacons. The larger the time between status update beacons, the lower the available reaction time. Figure 3 shows the surveillance strategy of the RCAS system. When the distance between the two trains is below $\mathrm{BC}$, the trains must brake, if they do not want to risk an accident. The status update beacons indicate to the trains the distance the other trains are. In the case no status update beacon is received between $\mathrm{TA}$ and $\mathrm{BC}$, the trains will not be aware of the traffic situation and will not be able to brake in time. Obviously, the time between the reception of consecutive status update beacons is a fundamental performance criterion in MAMNETs.

For example, in a MAMNET system with a beacon rate of $1 \mathrm{~Hz}$ (each node broadcasts a status update beacon every second), the failure in the reception of one status update beacon implies two seconds of update delay. For a node moving at $160 \mathrm{~km} / \mathrm{h}$, this means $90 \mathrm{~m}$ less to react in time before an accident might occur.

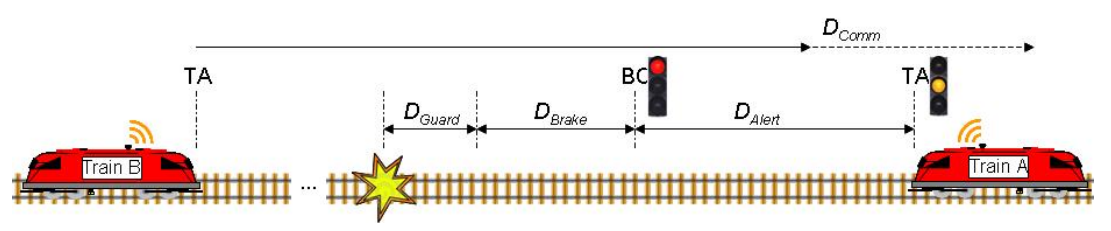

Fig. 3. RCAS surveillance strategy

We define the Update Delay as the time between the reception of two consecutive status update beacons coming from the same node. The Update Delay is the most relevant performance criterion in the MAC layer of the MAMNETs. Since the time between the transmission of two consecutive beacons, around 
$1 \mathrm{~Hz}$ depending on the application, is called frame, the Update Delay can be as well defined as the number of frames between two consecutive status update beacons coming from the same node. Some authors use related criteria in the analysis of MAC layers in MAMNETs: mean beacon transmission delay [14] or Maximum distance between two successful communications [15]. It is necessary to establish a general metric for the Update Delay criterion. This metric must give a complete description of the behavior of the Update Delay and should be application independent, i.e, should not depend on a concrete number of nodes in the MAMNET or a particular beacon rate. Both proposed metrics in [14] and [15] are not complete descriptions of the behavior of the Update Delay in the network, nor application independent.

We propose the Complementary Cumulative Distribution function (CCDF) of the Update Delay as the main metric for the performance of MAC layers in MAMNETs. This metric represents for each point the probability that the update delay $U D$ is larger than the number of frames $t f$ in the abscissa.

$$
C C D F(u d)=P(U D>u d=t f)
$$

Figure 4 shows an example of the CCDF of the Update Delay.

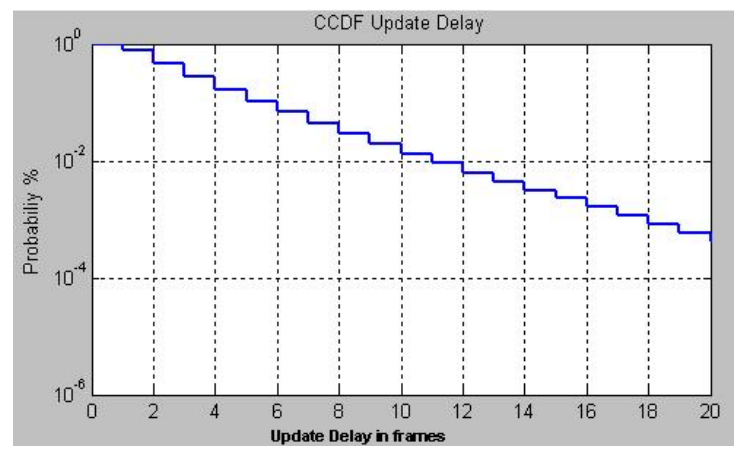

Fig. 4. Update delay metric: CCDF

The abscissa of the curve can be easily translated into seconds for a concrete system in the following way: $t=u d \cdot t f$ where $t$ is the update delay in seconds, $u d$ is the update delay in frames and $t f$ is the duration of a frame in seconds. For example for the Car2Car communication system $t f=0.5$ since the cars send an update beacon twice per second. This translation can be directly done when the propagation channel is an ideal channel. On the other case, the CCDF should be computed for different frame lengths.

Figures 5 and Figure 6 shows two examples of update delay CCDFs.

\subsection{Throughput}

The throughput is defined as the fraction of the capacity of the channel that is used. It indicates the efficiency of the MAC layer. The maximum throughput is 


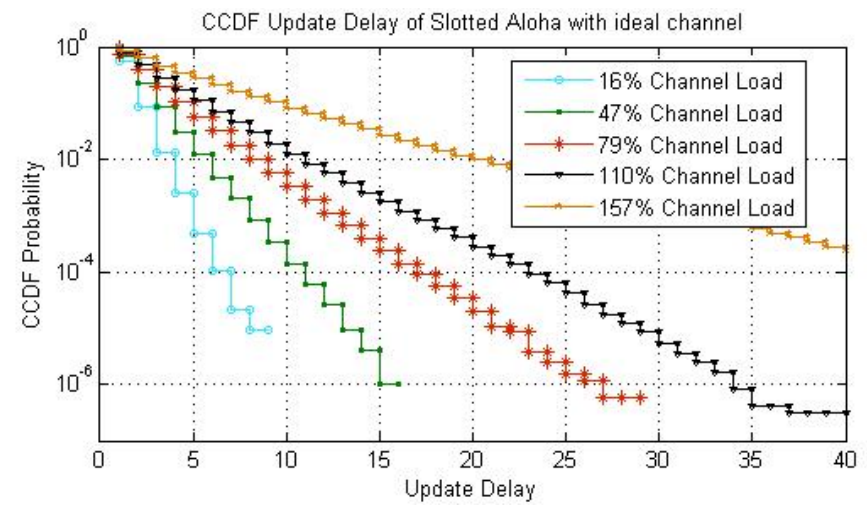

Fig. 5. CCDF of Slotted Aloha for different net channel loads

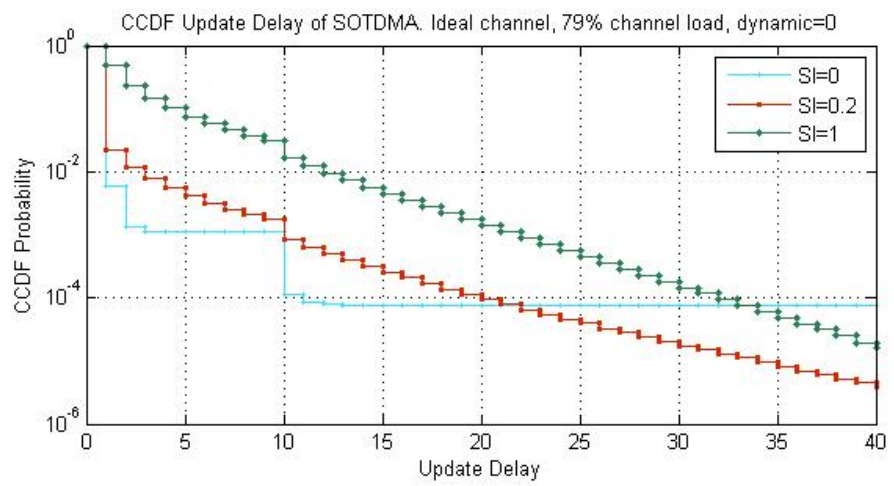

Fig. 6. CCDF of SOTDMA for different SI factors

achieved when the number of correctly delivered packets equals the number of packets for a $100 \%$ net channel load. Commonly, the MAC layers are not able to reach $100 \%$ throughput; there are two main reasons: Firstly, the MAC layer may add a header and waiting times so that the length of the packet is bigger than the real payload. Figure 7 shows this case. Secondly, due to collisions the number of correctly delivered packets cannot reach the number of packets for a $100 \%$ net channel load.

Channel load $=\mathbf{5 8} \%$

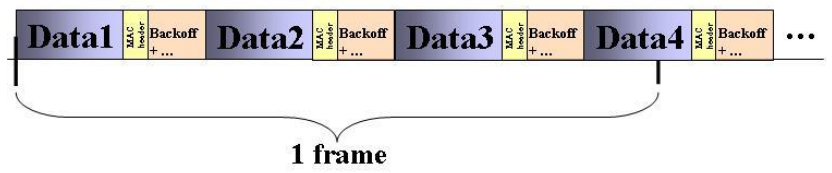

Fig. 7. A full channel that does not reach $100 \%$ throughput

In Figure 8 the throughput of slotted Aloha and SOTDMA under the same influence factors can be observed. 


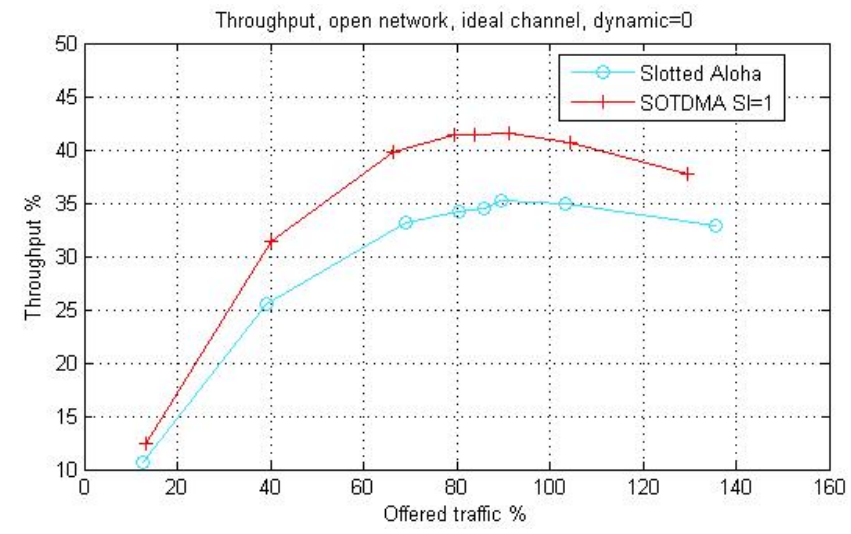

Fig. 8. Comparison of the throughput of Slotted Aloha and SOTDMA

\subsection{Collision rate}

The collision rate is defined as the colliding fraction of the transmitted messages [11]. There is a direct relation between the collision rate $C R$ and the throughput TH when the channel load is $100 \%$ : $C R_{\text {ChannelLoad } 100 \%}+T H_{\text {ChannelLoad } 100 \%}=$ 1.

Note that in a radiobroadcast network, every time a node transmits a message, it is received by many nodes in its surroundings. Therefore, to compute the number of delivered messages, the total amount of received messages should be divided by the total amount of nodes that should receive it, i.e. the nodes within the communication range.

Figure 9 shows the influence of the propagation channel on the collision rate of slotted aloha.

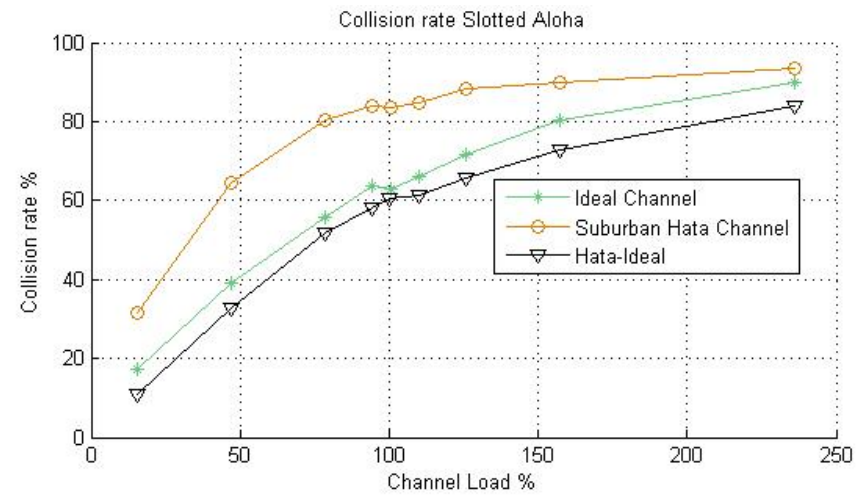

Fig. 9. Collision rate of Slotted Aloha for different propagation channels 


\section{Conclusions}

This paper has presented a road map to analyse the performance of the MAC layers in mobile ad-hoc multibroadcast networks. The particularities and challenges of this kind of networks have been introduced. We have identified the factors that influence the behaviour of the MAMNETs MAC layer protocols and finally the most relevant MAC performance metrics in MAMNETs have been studied.

\section{References}

1. N. Abramson. Computer Communication Networks. Prentice-Hall Inc., Englewood Cliffs, New Jersey, 1973.

2. ADS-B Home Page. http://adsb.tc.faa.gov/ads-b.html.

3. AIS official website. http://www.navcen.uscg.gov/enav/ais/default.html.

4. Cristina Rico García Andreas Lehner, Thomas Strang. Comb: Cell based orientation aware manet broadcast mac layer. In IEEE GLOBECOM 2008, 2008.

5. Achim Brakemeier. Network design limits and vanet performance. White Paper V0.5 (2008-11), Car 2 Car Communication Consortium, November 2008.

6. Car2Car communication consortium. http://www.car-to-car.org/.

7. Stephan Eichler. Performance evaluation of the IEEE 802.11p WAVE communication standard. In Proceedings of the 1st IEEE International Symposium on Wireless Vehicular Communications (WiVeC), September 2007.

8. Ajay C. Gummalla and John O. Limb. Wireless medium access control protocols. Communications Surveys $\&$ Tutorials, IEEE, 3(2):2-15, November 2009.

9. ITU. Itu-r m.1371* technical characteristics for a universal shipborne automatic identification system using time division multiple access in the vhf maritime mobile band, 2001.

10. Sunil Kumar, Vineet S. Raghavan, and Jing Deng. Medium access control protocols for ad hoc wireless networks: A survey. Ad Hoc Networks, Elsevier, 4(3):326-358, 2006.

11. Thierry Turletti Lamia Romdhani, Qiang Ni. Adaptive edcf: Enhanced service differentiation for ieee 802.11 wireless ad hoc networks. In IEEE W3reless Communications and Networking Conference (WCNC 2003), March 16-20, 2003.

12. Status of Project IEEE 802.11 Task Group . http://www.ieee802.org/11/reports/tgp-update.html.

13. Thomas Strang, Michael Meyer zu Hörste, and Xiaogang Gu. A railway collision avoidance system exploiting ad-hoc inter-vehicle communications and galileo. In 13th World Congress and Exhibition on Intelligent Transportation Systems and Services (ITS 2006), Oktober 2006.

14. Alexey Vinel, Yevgeni Koucheryavy, Sergey Andreev, and Dirk Staehle. Estimation of a successful beacon reception probability in vehicular ad-hoc networks. In IWCMC '09: Proceedings of the 2009 International Conference on Wireless Communications and Mobile Computing, pages 416-420, New York, NY, USA, 2009. ACM.

15. Junji Imai Noriyoshi Suzuki Yukihiro Tadokoro, Kenji Ito and Nobuo Itoh. A new approach for evaluation of vehicle safety communications with decentralized tdma-based mac protocol. In IEEE Intelligent Vehicle Symposium, 2008.

16. Hongqiang Zhai, Jianfeng Wang, Xiang Chen, and Yuguang Fang. Medium access control in mobile ad hoc networks: challenges and solutions: Research articles. Wirel. Commun. Mob. Comput., 6(2):151-170, 2006. 